J. Lake Sci.(湖泊科学), $2006, \mathbf{1 8}(6): 557-564$

http:// www. jlakes.org. E-mail: jlakes@niglas.ac. cn

(c) 2006 by Journal of Lake Sciences

\title{
磷循环的非线性动力学特性及富营养化
}

\author{
韦海英, 柴立和
}

(天津大学环境科学与工程学院, 天津 300072)

\begin{abstract}
摘 要: 本文在假设湖泊受单一营养盐磷限制和不考虑湖底沉积物影响的情形下, 建立了一个单层箱体磷循环的非线性 动力学模型, 研究湖泊中颗粒磷、可溶性磷和藻类中的磷这三种磷之间的相互关系, 理论上分析了磷循环系统的稳定特 性,得出三种形式的磷共存是系统趋向稳定的平衡点. 然后论文又从人工治理的角度, 通过数值计算综合考虑外源性磷、 水力冲刷、生物调控和基质浓度因素对磷循环的影响, 分析不同因素的改变对系统和湖泊水质的影响,进而对这一类的富 营养化现象防治提供可行性的工程上的实际建议.
\end{abstract}

关键词 : 磷循环; 非线性动力学;富营养化

\section{Non-linear dynamic characteristics of phosphorus cycle and eutrophication}

\section{WEI Haiying \& CHAI Lihe}

(School of Environmental Science and Engineering, Tianjin University, Tianjin 300072, P. R. China)

\begin{abstract}
Assumping the lake is P-limited and ignoring the effects of sediment, the paper proposes a one dimensional single-box phosphorus cycling model for the interactions among particulate phosphorus, dissolved reactive phosphorus and $\mathrm{P}$ in the alga. Nonlinear dynamics theory are used to investigate the model. Theoretical result shows that coexisting of three forms of phosphorus will dominate the dynamic trends of the system. For the sake of practical interests, numerical simulations are further carried out to reveal effects of the external phosphorus, hydraulic eroding, regulation of biology and nutrient concentrate on phosphorus cycling. The numerical results not only reveal how variations of different parameters influence the water quality of lakeg, but also shed new lights on industrial applications of controlling eutrophication.
\end{abstract}

Keywords: Phosphorus Cycle; non-linear dynamics; eutrophication

早在上个世纪八十年代初期, 丹麦著名生态学家 Jørgensen 曾指出浮游藻类的生长是富营养化的关键 过程, 所以研究营养物负荷和浮游藻类生产力的相互关系和作用, 是我们揭示富营养化形成机理的主要途 径. 长期以来碳、氮、磷被认为是引起水体富营养化的主要物质, 而通常情况下三种物质在淡水中的存在比 率为 $106: 16: 1^{[1]}$, 又根据国际经济合作和开发组织 (OECD) 的调查显示, 大约 $80 \%$ 的湖泊富营养化是受磷 元素的制约, 其余的 $20 \%$ 分别与氮元素和其他因素有关. 为了更好地描述这种受磷限制的湖泊动态过程及 生态变化, 自从上个世纪六、七十年代以来, 科学家们逐步应用数学建模的方法来揭示湖泊水质的动态变化 规律 ${ }^{[2-8]}$, 数学模型在考察、预测、模拟水体水质进程方面已经取得了鼓舞人心的成果 ${ }^{[9-14]}$. 不过湖泊水生 生态系统是一个相当复杂的开放系统, 各个子系统或各种影响因素之间存在着复杂的非线性相互关系, 系 统内部的这些非线性作用会极大地影响着磷系统循环的稳定性, 造成系统对影响因素的变化具有极度敏感 性. 现有的研究在揭示这些非线性相互作用和各种因素对磷系统循环的敏感影响等方面还极待深人. 为了 更好地理解系统的非线性相互作用和各种因素对磷系统循环的影响, 进而描述湖泊水质的动态过程, 本文 建立了相应的非线性动力学数学模型, 对系统的平衡状态和局部稳定状态及系统的动力学行为进行了理论

* 天津大学杰出人才引进基金 (W20201)、教育部留学回国人员科研启动基金 (413042) 和国家自然科学基金 (50406018)共同资助. 2005-12-20 收稿;2006-04-24 收修改稿. 韦海英,女,1979 年生, 硕士 ; E-mail: 1hchai@ tju. edu. cn. 
分析, 并结合数值模拟揭示了模型中一些重要参数对磷系统循环的影响, 对防治受磷限制的湖泊富营养化 措施进行了讨论.

\section{1 淡水生态系统磷循环机理及模型}

磷在水体中, 以含磷有机物 (如核酸、植酸及卵磷脂) 和无机磷化合物如磷酸钻、磷酸钠、磷酸镁、磷灰石 矿石以及还原态 $\mathrm{PH}_{3}$ 三种形态存在 ${ }^{[15]}$. 磷是浮游植物重要的营养元素, 然而植物和微生物不能直接利用 含磷有机物和不溶性的磷酸钙, 他们必须经过微生物分解转化为溶解性的磷酸盐才能被吸收利用. 而后植 物体又在微生物的作用下分解转化为溶解性的偏磷酸盐 $\left(\mathrm{HPO}_{4}^{2-}-\mathrm{P}\right), \mathrm{HPO}_{4}^{2-}-\mathrm{P}$ 在厌氧条件下被还原为 $\mathrm{PH}_{3}$, 以此构成磷的循环.

首先我们假设一个不分层的、浓度完全混合均匀的浅水湖泊, 它是一个主要以浮游藻类为生产力的水 生态系统, 并且这个系统应该是在有氧的状态下进行的, 同时我们还假设它是一个在一定条件下不受底泥 磷释放影响的系统, 这个磷循环系统如图 1 所示.

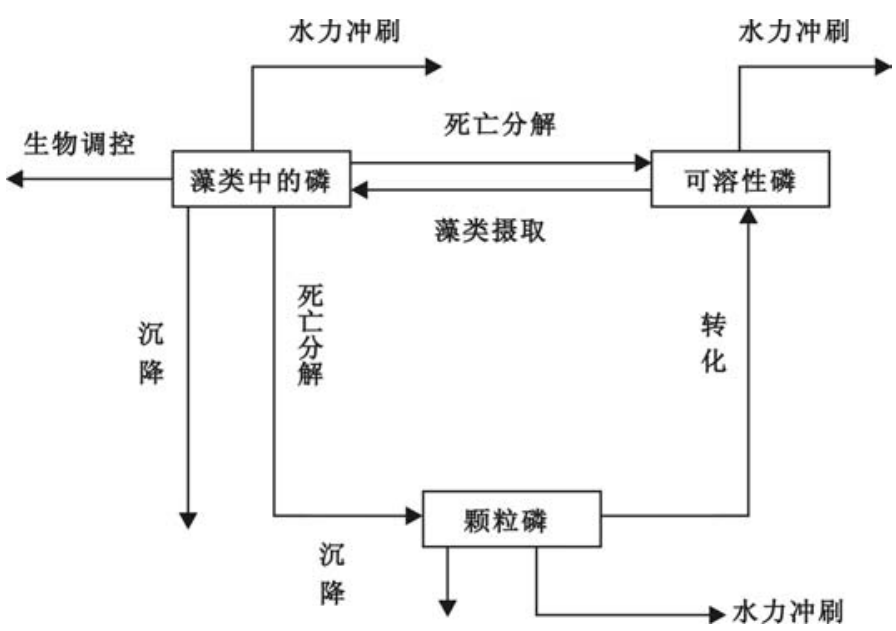

图 1 磷循环示意图

Fig. 1 Illustration of phosphorus cycles

根据磷在三种物质形态之间的转化, 可以建立一个以磷物质守恒的模型:

$$
\left.\begin{array}{l}
\mathrm{d} P_{A} / \mathrm{d} t=R_{\max } f(I) f(T) f\left(P_{S}\right) P_{A}-G_{P Z} P_{A}-D_{P L} P_{A}-K_{P A} P_{A}-\rho_{w} P_{A} \\
\mathrm{~d} P_{S} / \mathrm{d} t=L_{P S}+K_{Z} P_{P}+D_{P L} P_{A}\left(1-f_{O P}\right)-R_{\max } f(I) f(T) f\left(P_{S}\right) P_{A}-\rho_{w} P_{S} \\
\mathrm{~d} P_{P} / \mathrm{d} t=L_{P P}+D_{P L} P_{A} f_{O P}-K_{Z} P_{P}-K_{P P} P_{P}-\rho_{w} P_{P}
\end{array}\right\}
$$

上式中符号的意义分别为: $P_{A}$, 藻类中的磷浓度 $(\mathrm{mg} / \mathrm{L}) ; P_{S}$, 可溶性磷浓度 $(\mathrm{mg} / \mathrm{L}) ; P_{P}$, 颗粒磷浓度 $(\mathrm{mg} / \mathrm{L}) ; R_{\max }$, 浮游植物最大生长率; $f(I)$, 光消减系数; $f(T)$, 浮游植物最大生长率时的温度影响系数; $f\left(P_{S}\right)=P_{S} /\left(K_{m p}+P_{S}\right), K_{m p}$ 是水体中可溶性磷的半饱和常数; $G_{P Z}$, 生物调控影响参数(由于这个因素难 以确定, 本文用这个参数表示系统受其影响的大小); $D_{P L}$, 浮游植物死亡降解率; $K_{P A}$, 浮游植物沉降率, $K_{P A}=V_{P A} / H ; V_{P A}$, 浮游植物沉降速率; $H$, 湖体平均水深; $K_{Z}$, 颗粒磷转化成可溶性磷的比率; $f_{o p}$, 浮游植 物死亡降解转化成颗粒磷的百分数; $K_{P P}$, 颗粒磷的沉降率, $K_{P P}=V_{P P} / H, V_{P P}$, 颗粒磷的沉降速率; $\rho_{w}$, 水

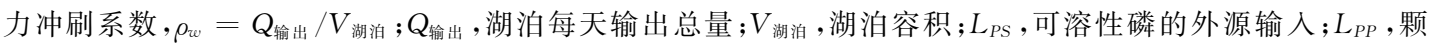
粒磷的外源输入.

\section{2 模型的理论分析}

下面对 (1) 式进行详细的数学分析, 令 (1) 式左边等于 0 , 可以得到两个非负平衡点: $E_{1}\left(0, P_{S} *, P_{P} *\right)$ 和 $E_{2}\left(P_{A^{\prime}}, P_{S^{\prime}}, P_{P^{\prime}}\right)$, 其中 $E_{1}$ 表示浮游植物的磷降为 0 , 即浮游植物物种消失, 这是一个特殊状态, 从模型的 
表达式上可以看出, 当所给的藻类中的浓度初值很低, 在系统运行的初期, 藻类还处于慢速生长阶段, 摄取 溶解性磷进行光合作用的速率小于它死亡分解、沉降和被水力冲刷的速率, 所以在前期阶段, 系统可能出现 了一个藻类中的磷消失的平衡点 $E_{1}$. 当系统中又不断输人外源性磷, 藻类得到充足的营养物质, 加上适宜 的温度和光照条件, 藻类很容易达到指数生长期, 这很容易使得系统偏离平衡点 $E_{1} . E_{2}$ 表示三种形态的磷 共存, 这在湖泊中是非常普遍的, 当三种形态的磷的制约关系达到平衡时, 三种磷浓度趋向于稳定的值. 一 个系统出现两个平衡点意味着系统原则上可以在两个平衡点之间任意运动, 不过系统的最终趋向却取决于 哪一个平衡点是稳定的, 下面对这两个平衡点的稳定性进行分析, 首先假设一个以 $P_{A}, P_{S}, P_{P}$ 为变量的 函数:

$$
\begin{aligned}
& f\left(P_{A}, P_{S}, P_{P}\right)=\left(R_{\max } f(T) f(I) f\left(P_{S}\right) P_{A}-D_{P L} P_{A}-G_{P Z} P_{A}-K_{P A} P_{A}-\rho_{w} P_{A}, L_{P S}+K_{Z} P_{P}+D_{P L} P_{A}\right. \\
& \left.\left(1-f_{O P}\right)-R_{\max } f(T) f\left(P_{S}\right) P_{A}-\rho_{W} P_{S}, L_{P P}+D_{P L} P_{A} f_{O P}-K_{Z} P_{P}-K_{P P} P_{P}-\rho_{W} P_{P}\right)
\end{aligned}
$$

方程 (1) 的 Jacobi 矩阵方程为

$$
|J|=\left[\begin{array}{lll}
a_{11} & a_{12} & a_{13} \\
a_{21} & a_{22} & a_{23} \\
a_{31} & a_{32} & a_{33}
\end{array}\right]
$$

其中各项的具体表达式为: $a_{11}=R_{\max } f(I) f(T) f\left(P_{S}\right)-D_{P L}-G_{P Z}-K_{P A}-\rho_{w}$

$$
\begin{aligned}
& a_{12}=R_{\max } f(I) f(T) P_{A} K_{m p} /\left(K_{m p}+P_{S}\right)^{2}, a_{13}=0 ; \\
& a_{21}=D_{P L}\left(1-f_{O P}\right)-R_{\max } f(I) f(T) f\left(P_{S}\right), \\
& a_{22}=R_{\max } f(I) f(T) P_{A} K_{m p} /\left(K_{m p}+P_{S}\right)^{2}, \\
& a_{23}=K_{Z}, a_{31}=D_{P L} f_{O P}, a_{32}=0, a_{33}=-\left(K_{Z}+K_{P P}+\rho_{w}\right) .
\end{aligned}
$$

代人平衡点 $E_{1}\left(0, P_{S} *, P_{P} *\right)$ 的具体值得:

$$
\begin{aligned}
& a_{11}=R_{\max } f(I) f(T) f\left(P_{S} *\right)-D_{P L}-G_{P Z}-K_{P A}-\rho_{w}, a_{12}=0, a_{13}=0, \\
& a_{21}=D_{P L}\left(1-f_{O P}\right)-R_{\max } f(I) f(T) f\left(P_{S} *\right), a_{22}=0, a_{32}=K_{Z}, a_{31}=D_{P L} f_{O P}, a_{32}=0, \\
& a_{33}=-\left(K_{Z}+K_{P P}+\rho_{w}\right)
\end{aligned}
$$

可得在平衡点 $E_{1}\left(0, P_{S} *, P_{P} *\right)$ 的 Jacobi 矩阵的三个特征根为:

$$
w_{1}=0, w_{2}=-\left(K_{Z}+K_{P P}+\rho_{w}\right), w_{3}=R_{\max } f(I) f(T) f\left(P_{S} *\right)-D_{P L}-G_{P Z}-K_{P A}-\rho_{w}
$$

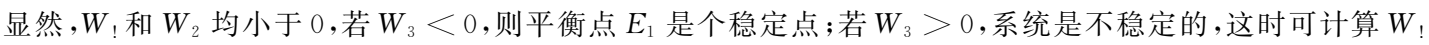
$+W_{2}+W_{3}$ 的值, 当其值大于 0 时, 相体积发散, 可判断属超混沌现象, 当其值小于 0 , 相体积收缩, 这是混沌 中奇怪吸引子的情形. 可见在 $W_{3}$ 的表达式中, 当具体参数值未定时, 这个平衡点的稳定性不能判断, 在下 文模型的数值计算中再具体讨论它的稳定性.

把平衡点 $E_{2} B\left(P_{A^{\prime}}, P_{S^{\prime}}, P_{P^{\prime}}\right)$ 的值代人上述 Jacobi 特征矩阵计算其特征值得到一个三次幂的特征方程,

$$
\lambda^{3}+C_{1} \lambda^{2}+C_{2} \lambda+C_{3}=0
$$

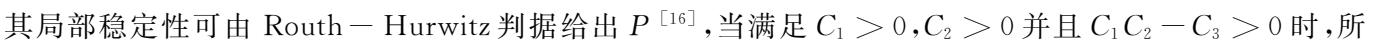
有特征值实部均为负值, 则局部是稳定的. $C_{1}, C_{2}, C_{3}$ 具有复杂的表达式, 同理当具体的参数值未定时, 这 个平衡点的稳定性不能判断, 仍在下文模型的数值计算中再具体讨论它的稳定性.

\section{3 模型的数值计算}

为了进一步详细阐述系统的动力学变化特性, 下面将采用数值方法在正实数域 $\mathrm{RP}+\mathrm{P}$ 上分析这个系 统的全局性行为. 在 (1) 式中得到系统的行为依赖于 12 参数, 如果考虑所有参数的变化, 不仅在数值计算上 比较困难而且对于分析湖泊磷循环系统的动力学行为的影响因素也没必要. 所以为了凸现问题的重点, 主

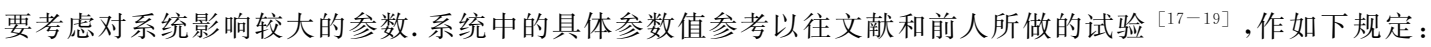
$R_{\max }=1.4-2.5 \mathrm{~d}^{-1} ; f(I)=0.95 ; f(T)=1.05 ; D_{P L}=0.15-0.35 \mathrm{~d}^{-1} ; G_{P Z}=0.1-1.0 \mathrm{~d}^{-1} ; V_{P A}=0.1$ $\mathrm{m} / \mathrm{d} ; V_{P P}=0.01-0.08 \mathrm{~m} / \mathrm{d} ; K_{Z}=0.15-0.30 \mathrm{~d}^{-1} ; f_{o p}=0.1-1.0 ; K_{m p}=0.0032-0.02 \mathrm{mg} / \mathrm{L}$.

为了对上文磷循环系统的理论分析做进一步论证, 分别做了一下计算和模拟, 先检验选定了具体参数 值时两个平衡点的稳定性, 在上述参数范围内选定两组典型参数值来计算模型的平衡点值和其特征值. 第 
一组典型参数值代人得两个平衡点为 $E_{1}(0,0.0379,0.0043)$ 和 $E_{2}(0.0128,0.0045,0.0092)$, 它们的特征值 分别为 $0,-0.7400,0.8396$ 和 $0,-0.7400,-0.0026$, 可见第一个为不稳定平衡点, 第二个稳定平衡点. 第 二组典型参数值代人计算得两个平衡点为 $\mathrm{E}_{1}(0,0.1978,0.0119)$ 和 $\mathrm{E}_{2}(0.0731,0.0026,0.0465)$, 它们的特 征值分别为 $0,-0.2700,1.9480$ 和 $0,-0.2700,-0.0016$, 所得结论同上.

下面给出两组具有同一组控制参数但赋予不同初始值 $P_{A}^{0}, P_{S}^{0}, P_{P}^{0}$ 的数值模拟的磷浓度时序列图 $2 \mathrm{a}$, 在图 $2 \mathrm{~b}$ 中, 假设湖泊处在营养物高浓度能够充分满足藻类的生长:
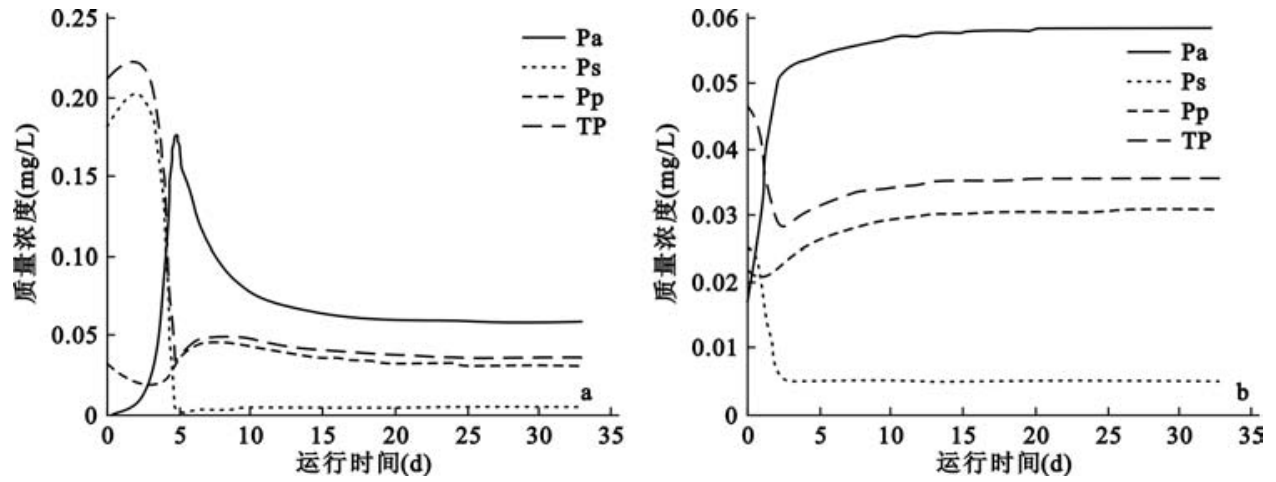

图 2 磷浓度随时间的变化

$\left(\mathrm{a}: P_{A}^{0}, P_{S}^{0}, P_{P}^{0}=0.00035 、 0.180 、 0.032 \mathrm{mg} / \mathrm{L} ; \mathrm{b}: P_{A}^{0}, P_{S}^{0}, P_{P}^{0}=0.0150 、 0.0250 、 0.0220 \mathrm{mg} / \mathrm{L}\right)$

Fig. 2 The variations of phosphorus concentrations with time

根据图 2 的结果, 同时结合上面的理论分析, 可以看出, 当藻类中的磷不存在时的平衡点是不稳定点, 在藻类中磷的浓度很低和营养物浓度高时易出现这个不稳定的平衡点, 但在系统内子系统间互相影响下, 它势必趋向稳定点即三种形式磷共存的稳定点, 在这个过程中, 控制好藻类的生长和总磷的浓度变化以避 免系统向富营养化发展是控制的关键.

下面我们将用太湖为实验对象做一个模拟, 以便验证这个模型对系统的趋势预测是可行的. 由于本文

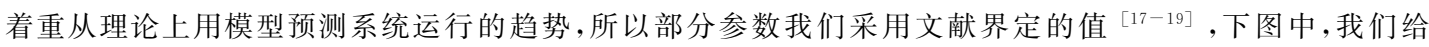
定: 借鉴文献 ${ }^{[20]}$ 的资料, 我们使用如下方法估算模型中的参数值: $L_{p p}$ 和 $L_{p s}$ 分别等于湖泊每天输人的量与 湖泊总容积之比; 水力冲刷系数等于湖泊每天输出水量与湖泊容积之比. 根据以上方法和文献所提供的数 据, 又因为据 30 年来对营养物的变化研究, 太湖有机物是总磷的主要成分, 其人湖量的增加是太湖近年来 总磷含量变幅增大的首要原因 ${ }^{[20]}$, 我们估略得到如下数值: $L_{P P}=0.0001 \mathrm{mg} / \mathrm{L}, L_{P S}=0.001 \mathrm{mg} / \mathrm{L}, \rho_{w}=$ 0.006. 资料显示, 太湖的浮游生物以铜绿微囊藻为优势种, 而这种藻类更容易利用水体中低浓度的磷, 与其 他藻类的竞争中占优势, 所以太湖水体中的微囊藻密度通常维持在较高水平. 假设在春夏季, 光照很充分, 温度适宜, 藻类疯狂繁殖生长, 依据文献 ${ }^{[20]}$, 和 $1 \mathrm{~g}$ 湿重藻类内约含 $0.0008 \mathrm{~g}$ 磷 ${ }^{[21]}$, 假定湖泊中藻类浓度 处在较高水平,而其他两种形式的磷处在低浓度时期,则三种形式的磷浓度分别为为 $0.0015 \mathrm{mg} / \mathrm{L} 、 0.0005$ $\mathrm{mg} / \mathrm{L}$ 和 $0.00008 \mathrm{mg} / \mathrm{L}$, 根据以上数据模拟结果见图 3 .

从图 3 的模拟结果来看, 模型反映了湖泊一定的动态运行趋势: 当湖泊中三种形式的磷都处于低浓度 时, 向湖泊中不断输人外源性磷, 藻类中磷在系统运行初始阶段, 由于可溶性磷的浓度很低, 藻类没有足够 的营养物吸收生长而使得藻类中的磷浓度下降, 当系统中不断输人外源磷, 藻类能够得到充足的营养物质 迅速生长, 使得藻类中的磷的浓度也迅速提高, 当湖泊中的磷达到一定阀值时, 浮游植物缓慢生长, 其生长 率和其他转化过程达到一定的平衡, 所以系统运行到一定阶段后, 藻类中的磷稳定在一定的高值上. 而可溶 性磷的浓度也是因为藻类的吸收利用, 在经过短期的迅速提高后, 又回降在一定值. 正是因为湖泊中的这种 内部动力学行为, 使得湖泊具有一定的自净能力, 但同时也可看到污染物的输人对湖泊内生态系统的变化 有很大的影响. 
从模型的表述来看, 这个系统的影响因素是很多 的, 其中气候环境的影响就是一个不可忽略的重要因 素, 但外部的气候条件往往是难于控制的, 所以本文主 要从人工治理可行性的角度上对以下因素进行讨论: 外 源性磷的输人、水力冲刷、生物调控以及基质浓度. 下文 的模拟是在图 2 的基础上进行的:

(1) 外源性磷输人大小的影响. 在模型中分别增大 $L_{P S}$ 和 $L_{P P}$ 的值可得到图 4 的浓度时序列图. 图 4 中由 于增大了外源性磷, 藻类中的磷浓度和总磷浓度也迅速 增大, 在系统逐渐达平衡时虽有所下降, 但仍持续在较 高浓度上. 可见,若不控制好外源性磷的输人,对于受磷 限制的湖泊, 其湖泊内的藻类易出现疯长现象, 这就有 可能使湖泊出现“水华”而导致富营养化的发生,并且大

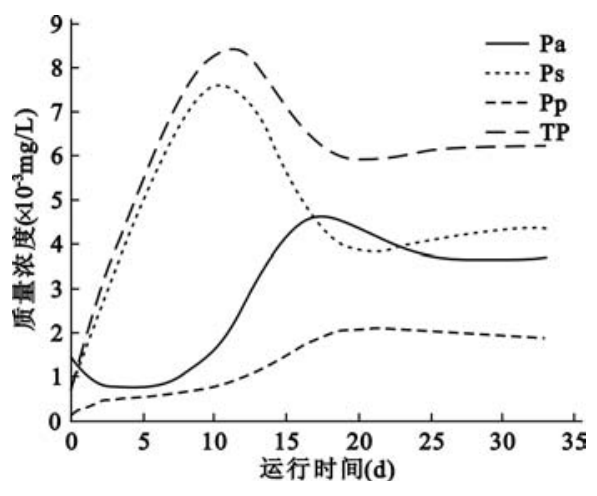

图 3 磷浓度随时间的变化 量外源性磷的输人也会对水体中的浮游动物甚至对以 $L_{p p}=0.0001 \mathrm{mg} / \mathrm{L}, L_{P S}=0.01 \mathrm{mg} / \mathrm{L}, \rho_{w}=0.006$ ) 湖泊水作为生活用水的周边人群产生危害. 大量的研究 和调查情况表明,绝大多数水体富营养化主要是外界输 Fig. 3 The variations of phosphorus concentrations with time 人的营养物质在水体中的富集造成的. 所以从长远的观 点来看,要想从根本上控制湖泊水体富营养化,首先应 该着重减少或者截断外部营养物质的输人,而其中点源的控制是我们控制的重点也是易控制部分. 荷兰从 1980 年开始通过国家和国际合作项目对其境内 231 个湖泊进行了十几年的营养负荷和改变富营养化状况 的研究,他们在 Naardermeer 自然保护区的浅湖采用无磷酸盐水降低外来营养负荷的办法,对控制富营养

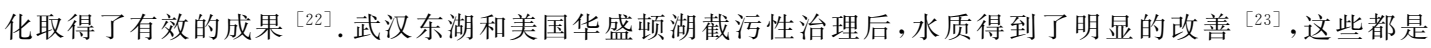
控制外源性磷输人的成功例子.
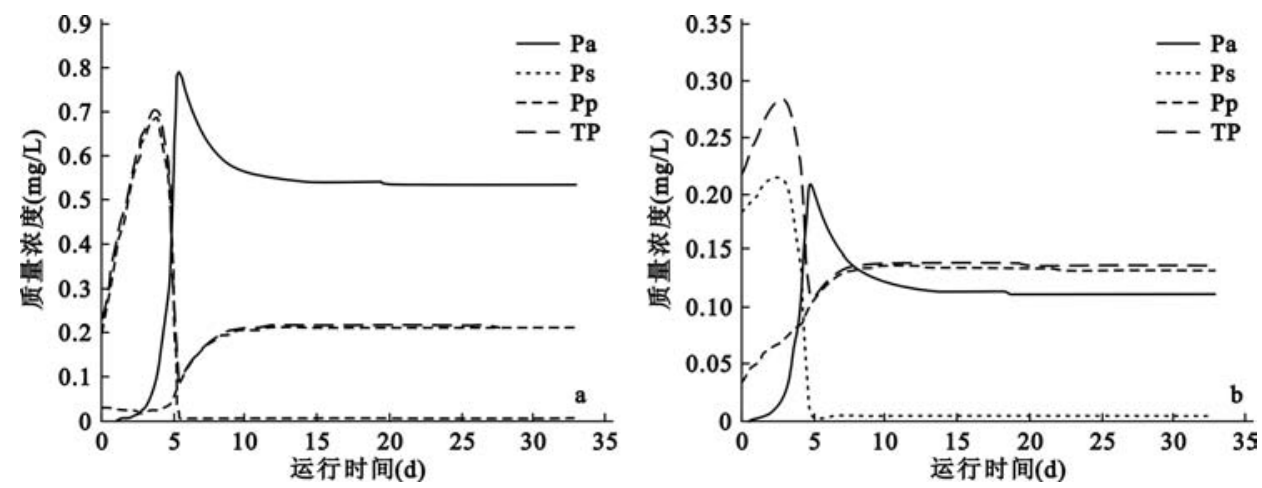

图 4 磷浓度随时间的变化

$$
\text { ( } \mathrm{a}: L_{P S}=0.180 \mathrm{mg} / \mathrm{L} ; \mathrm{b}: L_{P P}=0.032 \mathrm{mg} / \mathrm{L} \text { ) }
$$

Fig. 4 The variations of phosphorus concentrations with time

(2) 水力冲刷系数的影响. 在图 2 的基础上增大 $\rho_{w}$ 得到图 5 的时序列图, 从图 5 可以看出增大水力冲 刷系数后, 藻类中的磷和总磷的浓度都得到了很好的控制. 这是因为一方面可溶性磷和颗粒磷在大的水流 下易流出湖泊, 另一方面短时期的可溶性磷的存在不宜于浮游植物生长吸收, 这就抑制了藻类在适宜条件 下的疯狂生长, 因而增大湖泊水体流动性, 稀释营养物质浓度也是一种很好的控制方法. 这种控制措施降低 了营养物质的停留时间, 除了通过引水人湖的方式外, 还可采取迅速从集水处去除以及挖掘排水渠和阴沟 等的方法 ${ }^{[24]}$. 


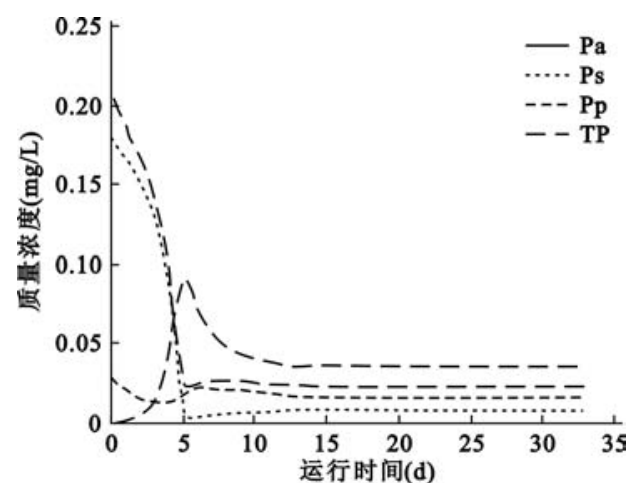

图 5 磷浓度随时间的变化 $\left(\rho_{w}=0.2\right)$

Fig. 5 The variations of phosphorus concentrations with time $\left(\rho_{w}=0.2\right)$

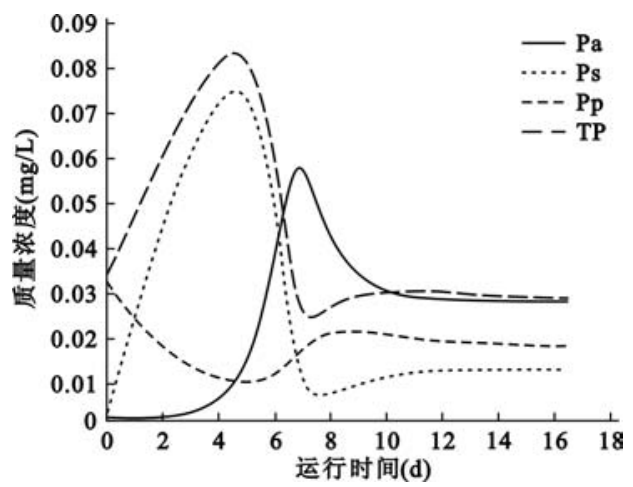

图 7 磷浓度随时间的变化

$$
\left(P_{S}^{0}=0.00030 \mathrm{mg} / \mathrm{L}\right)
$$

Fig. 7 The variations of phosphorus concentrations with time $\left(P_{S}^{0}=0.00030 \mathrm{mg} / \mathrm{L}\right)$

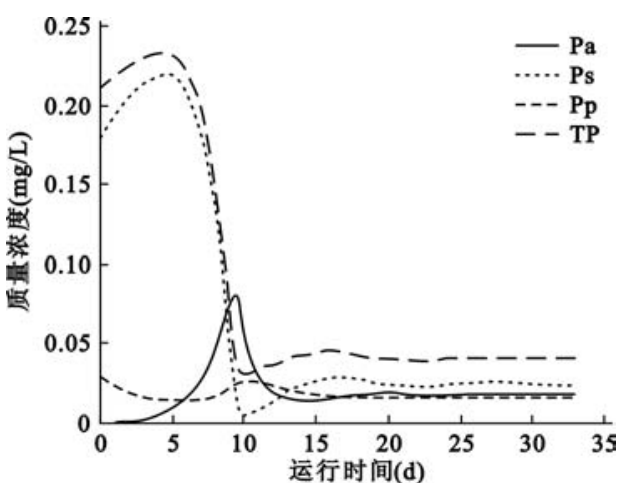

图 6 磷浓度随时间的变化 $\left(G_{P Z}=0.95\right)$

Fig. 6 The variations of phosphorus concentrations with time $\left(G_{P Z}=0.95\right)$

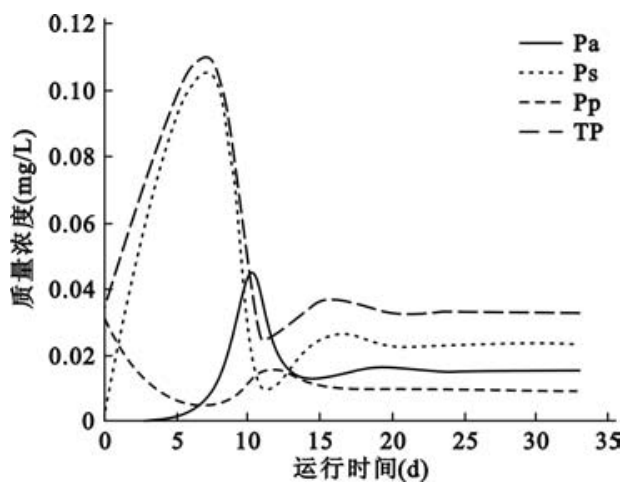

图 8 磷浓度随时间的变化 $\left(P_{S}^{0}=0.00030 \mathrm{mg} / \mathrm{L}, G_{P Z}=0.95\right)$

Fig. 8 The variations of phosphorus concentrations with time $\left(P_{S}^{0}=0.00030 \mathrm{mg} / \mathrm{L}, G_{P Z}=0.95\right)$

(3)生物调控的影响. 在实际富营养化处理中,这是一种用生物调控取代营养控制的一种调控技术, 它 主要通过重建生物群落、减少藻类生物量、保持水面清澈度并提高生物多样性来实现生物调控目的, 可施行 的方法有放养控制藻类生长的浮游动物, 或种植能与藻类竞争光源和营养盐的水生植物. 由于生物调控的 复杂性, 本文为了直观性地说明, 在文中只用参数 $G_{P Z}$ 来表述, 图 6 显示在增大生物调控参数后, 藻类中的 磷明显降低. 这种控制方法现已成为国际上的研究热点, 在美国、荷兰、德国等地区, 研究者们做了很多实地 试验进行验证并取得了很大成功 ${ }^{[25-27]}$.

(4) 基质浓度的影响. 在其它参数不变的情况下,减小水体中可溶性磷的浓度得到图 7 的模拟结果,结 果显示澡类生长相对图 2 变得缓慢, 图 2 中藻类的磷在不到 $5 \mathrm{~d}$ 达最大值, 而在图 6 中约需 $7 \mathrm{~d}$. 说明基质浓 度对藻类生长有很大的限制作用. 若在低基质浓度的条件下, 再增大生物调控系数到 0.95 , 与图 7 比较, 图 8 的模拟结果表明, 在低基质浓度条件下, 藻类生长更易抑制. 如果再在低基质浓度条件下模拟以上的参数 条件,同样也可得到类似的结论.

上文提到的这几个控制参数在湖泊治理中根据实际情况都是可以互相交叉使用的, 许多湖泊富营养化 
治理成功的实例也都是多项措施的结合 ${ }^{[28,29]}$.

\section{4 结论}

本文建立湖泊水体中三种形式磷相互转化的数学模型, 运用非线性动力学方法进行理论分析, 揭示了 这个复杂非线性系统的运行状态和局部稳定性, 得到只有三种形式磷共存时才是系统的稳定的平衡点, 控 制好系统由不稳定到稳定的过程是我们要关注的重点. 同时, 本文又从人工治理可行性的基础上对一些影 响因素做了数值模拟和讨论, 以期给湖泊治理提供一些理论依据: 外源性磷是对控制受磷限制的湖泊的治 理基础; 通过增大水力冲刷从而降低营养物质的停留时间可有效控制藻类的生长和总磷浓度; 生物调控已 成为国际研究热点, 其成效已在许多实地试验中得到证实; 最后本文指出, 基质浓度也是需要重视的影响因 素, 低的基质浓度抑制藻类的生长, 并且使其它的湖泊治理措施更具有可行性. 由于本文是在非线性动力学 的理论方法结合数值模拟的基础上进行研究, 仅以给湖泊水质动态运行进行系统的预测和湖泊治理措施提 供可行的依据, 也对将来新的湖泊水质控制方法提供一些理论基础, 但湖泊水质系统运行的可操作性和其 内部机理还需要更深一步地研究和证实.

\section{5 参考文献}

[1] 张思仁, 张 经. 三峡水库对长江 N、P 营养盐截留效应的模型分析. 湖泊科学, 2003, 15(1): 41-47.

[2] Vollenweider R A. Input-output models with special reference to the phosphorus loading concept in limnology. Schweizeriche Zeitschrift fur Hydrologie, 1975,37: 53-83.

[3] Zorzen M W, Smith O J and Kimmel L V. A long-term phosphorus model for lakes: Application to Lake Washington, Modeling Biochemical Process in Aquatic Ecosystem. RP Canada, Ann Arbor, MI: Ann Arbor Science, 1976: 75-92.

[4] Kamp N L. Modeling the temporal variation in sediment phosphorus fractions. Interaction Between Sediments and Fresh Water, 1978: 277-285.

[5] Steven C C. Long-term phonomenological model of phosphorus and oxygen for stratified lakes. Water Research, 1991, 25(6): 707-715.

[6] J $\varphi$ gensen S E. A eutrophication model for a lake. Ecol Model, 1976, 2: 147-165.

[7] Cerco C F and Cole F. Three dimensional eutrophication model of chesapeake bay. Journal of Environment Engineering, 1993, 19(6): 1006-1025.

[8] Pilar H, Robert B A and Daniel P. Modeling eutrophication kinetics in reservoir microcosms. Water Research, 1997, 31(9): 2511-2519.

[9] Thomann R V,Segna J S. Dynamic Phytoplankton-phosphorus Model of Lake Ontario: ten-year verification and simulation. In phosphorus Management Strategies for Lakes. Ann Arbor Science, $1980: 153$ -190 .

[10] Hellstrom T. The effect of resuspension on algal production in a shallow Lake. Hydrobiologia, 1991, 213 : $183-190$.

[11] Maceina M J, Soballe D M. Wind-related limnological variation in Lake Okeechobee. Florida. Lake Resevoir Mgmt, 1990,6:93-100.

[12] Bhogal A, Young S D, Ralph R, et al. Modelling the residual effect of phosphate fertilizer in the Ropsley (UK) field triel 1978-1990. Fertilizer Research, 1996, 44(1) :27-36.

[13] Hakanson L, Ostapenia A P, Boulion V V. A mass-balance model for phosphorus in Lakes accounticy for biouptake and retention in biota. Freshwater Biology, 2003:48(5):928-950.

[14] Litwack H S, Dilorenz J L, Huang P, et al. Development of a simple phosphorus model for a large urban watersshed: A case study. Journal of Environmental Engineering-ASCE, 2006, 132(4): 538 -546 . 
[15] 娄金生,谢水波,何少华等. 生物脱氮除磷原理与应用. 长沙: 国防科技大学出版社, 2002:10.

[16] Nicolis G, Prigogine I. Self-organization in non-equilibrium system. New York: Wiley, 1997:70-89.

[17] Ambrose J r, Connolly J P, et al. WASP4, A Hydrodynamic and Water Quality Model-Model Theory. User's Manual and Programmer's Guider. U. S. Environment Protection Agency. Environmental Research Laboratory. Athens Georgia. EPA 1988:600/3-87/039.

[18] Bowie G L, Mills WB, et al. Rates, Constants, and Kinetics Formulations on Surface Water Quality Modeling (2nd edn). U. S. Environmental Protection Agency. Environmental Reseach Laboratory. Athens. Georgia. EPA/1985:600/3-85/040.

[19] Herman G, Mishri A, and Berman T. A long-term prediction model for totel phosphorus concentration in Lake Kinneret. Water Research, 1989, 23: 61-66.

[20] 黄渏平. 太湖水环境及其污染控制. 北京:科学出版社,2001:60-65,203,246-248.

[21] 杨戈逊 S E 著. 陆健健, 周玉丽 译. 生态模型法原理. 上海: 上海翻译出版公司, 1988: 234 .

[22] Bootsma M C, Barendregt A, Van Alphen J C A. Effectiveness of reducing external nutrient load entering a eutrophicated shallow lake ecosystem in the Naardormeer nature reserve, The Netherlands. Biological Conservation, 1999,90:197-201.

[23] 彭近新, 陈慧君. 水质富营养化与防治. 北京: 中国环境科学出版社, 1988:149-152.

[24] Jeppesen E, Sondrgaard, et al. Lake and catchment management in Denmark. Hydrobiologia, 1999, $396: 419-432$.

[25] Carpenter S R, Cole K L, et al. Biological-control of eutrophication in lakes. Environ Sci Technol, $1995,29: 784-786$.

[26] Hosper S H. Stable states, buffers and switches: An ecosystem approach to the restoration and management of shallow lakes in The Neterlands. Water Science \& Technology, 1998,37:151-164.

[27] Deppe T, Ockenfeld K, Meybohm A, Opitz M, Benndorf J. Reduction of Microcystis blooms in a hypertrophic reservoir by a combined ecotechnological strategy. Hydrobiologia, 1999, 409:31-38.

[28] Gulati R D, Van Donk E. Lakes in the Netherlands, their origin, eutrophication and restoration: stateof-the art review. Hydrobiologia, 2002, 478(1-3):73-106.

[29] Hukanson Lar Ostapenia, Alexander Parparov Arkady, et al. Management criteria for lake ecosystem applied to case studies of changes in nutrient loading and climate change. Lake \& Reservoirs Research and Management, 2003, 8(2):141-155. 